\title{
Attitude towards Vaccination among Health Science Students before the COVID-19 Pandemic
}

\author{
Pérez-Rivas Francisco Javier 1,*(D), Del Gallego-Lastra Ramón ${ }^{1}$, Esteban-Garcimartín Ana ${ }^{2}$, \\ Marques-Vieira Cristina Maria Alves ${ }^{3}$ (D) and Ajejas Bazán María Julia 1,4 (D)
}

1 Departamento de Enfermería, Facultad de Enfermería, Fisioterapia y Podología, Universidad Complutense de Madrid, Plaza Ramón y Cajal n 3, Ciudad Universitaria, 28040 Madrid, Spain; rgallego@ucm.es (D.G.-L.R.); majejas@ucm.es (A.B.M.J.)

2 Estudiante de Grado de Enfermería, Facultad de Enfermería, Fisioterapia y Podología, Universidad Complutense de Madrid, 28040 Madrid, Spain; aneste01@ucm.es

3 Center Interdisciplinary Research in Health, Institute of Health Sciences, Nursing School (Lisbon), Universidade Católica Portuguesa, 1649-023 Lisbon, Portugal; cristina_marques@ucp.pt

4 Academia Central de la Defensa, Escuela Militar de Sanidad, Ministerio de Defensa, 28040 Madrid, Spain

* Correspondence: frjperez@ucm.es

check for updates

Citation: Javier, P.-R.F.; Ramón, D.G.-L.; Ana, E.-G.; Alves, M.-V.C.M.; Julia, A.B.M. Attitude towards Vaccination among Health Science Students before the COVID-19 Pandemic. Vaccines 2021, 9, 644 . https://doi.org/10.3390/ vaccines 9060644

Academic Editors: Pedro Plans-Rubió and Ralph A. Tripp

Received: 8 May 2021

Accepted: 8 June 2021

Published: 12 June 2021

Publisher's Note: MDPI stays neutral with regard to jurisdictional claims in published maps and institutional affiliations.

Copyright: (c) 2021 by the authors. Licensee MDPI, Basel, Switzerland. This article is an open access article distributed under the terms and conditions of the Creative Commons Attribution (CC BY) license (https:// creativecommons.org/licenses/by/ $4.0 /)$.

\begin{abstract}
Health science students are tomorrow's health professionals, the duties of whom could include vaccination. This work examines the general attitude towards vaccination in students attending the Faculty of Nursing, Physiotherapy and Chiropody at a university in Madrid, Spain, using the 'Attitudes and Behaviour With Regard To Vaccination Among Health Science Students Questionnaire'. The results were subjected to multivariate analysis to identify the influence of sex, the degree being pursued, and 'course year'. The number of students vaccinated against influenza in the campaign preceding the present study was also recorded, as were the factors that influenced decision-making in this regard. A total of 934 students completed the questionnaire. Their beliefs regarding vaccination were positive (mean score 3.23 points out of 4 ), as was their behaviour (3.35/4). Their general attitude (all variables taken together) was therefore also good (3.27/4). Only 26.8\% of the students had been vaccinated against influenza. Beliefs scores among the students of nursing in their more senior course years were significantly better than those recorded for all other groups. These students also showed the best general attitude towards vaccination and formed the largest group vaccinated against influenza. The results obtained are encouraging since nursing students are the most likely of future healthcare professionals to be involved in vaccination programmes.
\end{abstract}

Keywords: attitudes; beliefs; health occupations; influenza vaccination; students; vaccination

\section{Introduction}

Vaccination is one of the most efficient public health interventions for controlling transmissible disease. Over recent decades systematic vaccination programmes have drastically reduced the mortality and morbidity associated with infectious disease [1], as well as the healthcare costs they incur [2].

Even though vaccination might be considered the all-time most efficient means of preventing disease [3] (with the exception of the provision of clean drinking water), and while vaccination coverage is very high in Europe, including Spain [4], recent years have seen the rise of anti-vaxxer movements which have led to an increasing rejection of, or at least hesitancy towards, vaccination [5,6].

The World Health Organisation's Strategic Advisory Group of Experts on Immunisation defines vaccine hesitancy as "a delay in accepting or rejecting safe vaccines, despite the availability of vaccination services" [7]. This hesitancy is influenced by factors such as complacency, convenience, and confidence. While complacency and convenience are related to the perception of risk of disease and accessibility to vaccination services, confi- 
dence is defined as trust in the safety and effectiveness of vaccines and the health system that delivers them [6].

The idea that vaccines are not safe, that they contain dangerous adjuvants, or that they might have unknown long-term adverse effects has contributed towards vaccine hesitancy. Such beliefs lead to people overestimating the risks and underestimating the benefits of vaccination [8]. Unfortunately, they are not limited to the general population; certain healthcare professionals also hold them [9]. This alarming situation has led the WHO to declare vaccine hesitancy as one of the top 10 threats to world health [10].

The role played by healthcare professionals with respect to vaccine hesitancy is crucial $[11,12]$. Healthcare workers are usually the main source of information for patients regarding health matters, including vaccination, and usually enjoy their trust $[13,14]$. The attitude of the former towards recommending vaccination is therefore vital. The 2007 Summit of Independent European Vaccination Experts (SIEVE) [15] made it clear that strategies aimed at optimising vaccination coverage among adults and children in Europe ought to be directed towards healthcare professionals, focusing on their attitude towards vaccination since this could determine the vaccination-related decisions made by families under their care [9]. Despite these recommendations, however, several studies have shown that many such professionals are worried about the safety of vaccines $[16,17]$, while others may find it difficult to respond to hesitant patients' questions because they themselves are hesitant $[18,19]$. These findings are worrying since such negative attitudes could influence the decisions taken by patients regarding vaccination $[11,14,16,20,21]$.

Health science students are tomorrow's healthcare professionals, and the responsibility of making vaccination programmes effective will eventually fall to them. Knowing their general attitude towards vaccination is therefore important, as is implementing any intervention required to modify their attitude should this be necessary. The aims of the present work were (1) to determine their beliefs regarding vaccination, their behaviour regarding vaccination (in terms of auto-recommendation of vaccination and recommending it to others, etc.), and their general attitude (all variables scores taken as a whole) with regard to vaccination, (2) to determine whether any differences exist between students of nursing, chiropody and physiotherapy in these respects, (3) to determine whether scores for these variables change as their courses progress, and (4) to identify whether any of these variables affect their seeking vaccination against influenza.

\section{Materials and Methods}

This cross-sectional study involved 934 students, all attending the Faculty of Nursing Physiotherapy and Chiropody, Universidad Complutense de Madrid (Spain), who responded to the 'Attitudes and Behaviour With Regard To Vaccination Among Health Science Students Questionnaire' (ACVECS according to its Spanish initials) [22]. Sample size requirement analysis showed 310 respondents were needed for a 5\% error and $95 \%$ confidence limits. All responders gave their informed consent to be included.

The ACVECS questionnaire examines beliefs, behaviour and general attitude towards vaccination. It is composed of 24 items; the first 15 of which examine beliefs, while the last 9 examine behaviour. Taken together, these 24 items determine the "general attitude" towards vaccination. All items were answered on a five-point Likert scale from $0=$ totally disagree, to $4=$ totally agree. For items 1,2, 7, 8, 15 and 23, the scores have to be inverted before analysis given the way in which these questions are phrased.

The researchers contacted the coordinators of the Nursing, Physiotherapy and Chiropody degree courses in order to organise a meeting with the students present on different days between 15-30 January 2020. During this meeting the study was explained, and it was made clear that anonymity was guaranteed at all times (names were neither required nor requested). It was hoped that this would also safeguard against bias in the students' answers caused through a desire to please their peers or teachers.

The students who wished to complete the questionnaire did so in situ over a period of 10-15 min, using their smartphones to access the questionnaire via the University's virtual 
campus. It they were unable to log in they were allowed to complete the questionnaire later from another physical location. The questionnaire was completed using an on-line Google Forms ${ }^{\circledR}$ form, which also collected information on respondent age, sex, degree being pursued, course year, and on whether the respondent had been vaccinated against influenza in the campaign preceding the study.

All information collected was transferred to a database designed using Microsoft Office Excel 2016. Beliefs, behaviour and general attitude scores of $\geq 3$ were considered positive (i.e., vaccination-favourable), $\leq 1$ negative and $=2$ considered neutral or indifferent.

The examined variables were first subjected to simple statistical description. The Kolmogorov-Smirnov test was used to examine the normality of distribution of the results. Quantitative variables were described as means \pm standard deviation (SD) (normally distributed results) or median and interquartile range (not normally distributed). The Chi squared test was used to compare qualitative variables, and the Student $t$ test or ANOVA to compare quantitative variables. Significance was set at $p<0.05 ; 95 \%$ confidence intervals $(95 \% \mathrm{CI})$ were also determined. The effect of sex, age, the degree being pursued, and course year (1st, 2nd, 3rd or 4th) on beliefs, behaviour and general attitude was examined by regression analysis. In addition, associations were also sought between all the studied variables and whether the students had been vaccinated against influenza in the campaign preceding the study period. Those variables that showed a significant relationship in bivariate analysis, or that were considered important even though they showed no association, were included in binary logistic regression and multiple linear regression analyses. The relationships between dependent and independent variables in binary logistic regression analyses were determined via the $p$ value and $95 \% \mathrm{CI}$ of the exponents of the $B$ coefficient $\left(e^{B}=O R\right)$.

All analyses were undertaken using the Statistical Package for the Social Sciences (SPSS) v.25 for Windows (IBMC).

The study was approved by the Research Committee of the Faculty of Nursing, Physiotherapy and Chiropody, Universidad Complutense de Madrid, and by the Ethics in Research Committee of the Hospital Universitario Clínico San Carlos. All work was performed in adherence to the principles of the Declaration of Helsinki (2013 version). All data were treated adhering to General Data Protection Regulation (GDPR) 2016/679, 27th April 2016, and the Spanish Ley orgánica de protección de datos y garantía de derechos digitales (LOPDGDD; Data Protection And Guarantee Of Digital Rights) 3/2018, 5 December.

\section{Results}

A total of 934 students responded to the questionnaire. This figure represents $59.1 \%$ of the entire student body attending the faculty $(65.6 \%$ of whom are nursing students, $16.6 \%$ physiotherapy students, and $17.8 \%$ chiropody students) (Table 1 ). Of those who responded, 31.2\% were in their first year, 32.1\% were in their second, $19.2 \%$ in their third, and $17.5 \%$ in their fourth. At least 30 students from each degree and course year enrolled, except for the third year of chiropody $(n=28)$. In this case, $79.5 \%$ of the responders were female. $66.9 \%$ of the respondents were students of nursing, $17.3 \%$ of physiotherapy and $15.8 \%$ of chiropody, reflecting well the distribution for the faculty as a whole (Table 2). The mean age of the respondents was $21.3 \pm 0.34$ years; the mean age of the chiropody students $(22.5 \pm 0.4$ years) was a little higher than that of the nursing $(21.1 \pm 5.4$ years $)$ and physiotherapy students $(20.6 \pm 0.24$ years $)(p<0.01)$.

Table 1. Participation in the study by degree being pursued and course year.

\begin{tabular}{|c|c|c|c|c|c|c|}
\hline \multirow{3}{*}{ Degree } & \multirow{3}{*}{$\begin{array}{c}\text { Total Matriculated } \\
n(\%)\end{array}$} & \multirow{3}{*}{$\begin{array}{c}\begin{array}{c}\text { Total Participating } \\
\text { Subjects }\end{array} \\
n(\%)\end{array}$} & \multicolumn{4}{|c|}{ Participating Subjects per Year } \\
\hline & & & 1 & 2 & 3 & $4^{\circ}$ \\
\hline & & & $n(\%)$ & $n(\%)$ & $n(\%)$ & $n(\%)$ \\
\hline Nursing & 1039 (65.6) & $624(66.9)$ & $220(35.2)$ & $192(30.8)$ & $120(19.3)$ & $92(14.7)$ \\
\hline Physiotherapy & $262(16.6)$ & $162(17.3)$ & $40(24.6)$ & $50(30.8)$ & $30(18.5)$ & $42(25.9)$ \\
\hline Chiropody & $281(17.8)$ & $148(15.8)$ & $32(21.6)$ & $58(39.2)$ & $28(18.9)$ & $30(20.3)$ \\
\hline TOTAL & $1582(100.0)$ & $934(100.0)$ & $292(31.2)$ & $300(32.1)$ & $178(19.2)$ & $164(17.5)$ \\
\hline
\end{tabular}


Table 2. Distribution of participating subjects by sex and course year in each degree pursued.

\begin{tabular}{|c|c|c|c|c|c|c|c|c|c|c|}
\hline \multirow{2}{*}{ Variables } & & \multicolumn{2}{|c|}{ Total } & \multicolumn{2}{|c|}{ Nursing } & \multicolumn{2}{|c|}{ Physiotherapy } & \multicolumn{2}{|c|}{ Chiropody } & \multirow{2}{*}{$p$ Value } \\
\hline & & $n(\%)$ & $95 \% \mathrm{CI}$ & $n(\%)$ & $95 \% \mathrm{CI}$ & $n(\%)$ & $95 \% \mathrm{CI}$ & $n(\%)$ & $95 \% \mathrm{CI}$ & \\
\hline \multirow{2}{*}{ Sex } & Female & $\begin{array}{c}743 \\
(79.5)\end{array}$ & $(77.0-82.1)$ & $\begin{array}{c}532 \\
(85.3)\end{array}$ & (83.0-87.6) & $\begin{array}{c}101 \\
(62.3)\end{array}$ & $(59.2-65.4)$ & $\begin{array}{c}110 \\
(74.3)\end{array}$ & $(71.5-77.1)$ & \multirow{2}{*}{$p<0.01$} \\
\hline & Male & $\begin{array}{c}191 \\
(20.5) \\
\end{array}$ & $(17.9-23.2)$ & $\begin{array}{c}92 \\
(14.7) \\
\end{array}$ & $(12.4-17.0)$ & $\begin{array}{c}61 \\
(37.7) \\
\end{array}$ & $(34.6-40.8)$ & $\begin{array}{c}38 \\
(25.7) \\
\end{array}$ & (22.9-28.5) & \\
\hline \multirow{4}{*}{$\begin{array}{c}\text { Course } \\
\text { year }\end{array}$} & 1 st & $\begin{array}{c}292 \\
(31.2)\end{array}$ & $(28.2-34.2)$ & $\begin{array}{c}220 \\
(35.3)\end{array}$ & $(32.2-38.4)$ & $\begin{array}{c}40 \\
(24.7)\end{array}$ & (21.9-27.5) & $\begin{array}{c}32 \\
(21.6)\end{array}$ & $(19.0-24.2)$ & \multirow{4}{*}{0.062} \\
\hline & 2nd & $\begin{array}{c}300 \\
(32.2)\end{array}$ & $(29.1-35.1)$ & $\begin{array}{c}192 \\
(30.8)\end{array}$ & $(27.8-33.8)$ & $\begin{array}{c}50 \\
(30,9)\end{array}$ & $(27.9-33.9)$ & $\begin{array}{c}58 \\
(39.2)\end{array}$ & $(36.1-42.3)$ & \\
\hline & $3 r d$ & $\begin{array}{c}178 \\
(19.0)\end{array}$ & $(16.5-21.5)$ & $\begin{array}{c}120 \\
(19.2)\end{array}$ & $(16.7-21.7)$ & $\begin{array}{c}30 \\
(18.5)\end{array}$ & $(16.0-21.0)$ & $\begin{array}{c}28 \\
(18.9)\end{array}$ & $(16.4-21.4)$ & \\
\hline & 4 th & $\begin{array}{c}164 \\
(17.6)\end{array}$ & $(15.1-19.9)$ & $\begin{array}{c}92 \\
(14.7)\end{array}$ & $(12.4-17.0)$ & $\begin{array}{c}42 \\
(25.9)\end{array}$ & $(23.1-28.7)$ & $\begin{array}{c}30 \\
(20.3)\end{array}$ & $(17.7-22.9)$ & \\
\hline
\end{tabular}

As a whole, the responding students scored a mean 3.23 (out of 4 ) in terms of belief, 3.35 in behaviour, and 3.27 in general attitude. Table 3 shows the distribution of these three dimensions with respect to sex, the degree being pursued, and course year. Female students obtained better beliefs, behaviour and general attitude scores than their male colleagues $(p<0.05)$. The students of nursing returned the highest scores for all three dimensions $(p<0.01)$. Moreover, their belief and general attitude scores significantly improved year on year as they progressed through their course $(p<0.05)$. The belief scores for the physiotherapy students improved, but not homogenously year on year.

Table 3. Distribution of scores for ACVECS questionnaire dimensions with respect to sex, degree being pursued and course year. Madrid, Spain, 2020.

\begin{tabular}{|c|c|c|c|c|c|c|c|}
\hline Variables & Categories & $\begin{array}{c}\text { Beliefs } \\
\text { Mean } \pm \text { SD }\end{array}$ & $p$ Value & $\begin{array}{c}\text { Behaviour } \\
\text { Mean } \pm \text { SD }\end{array}$ & $p$ Value & $\begin{array}{c}\text { General } \\
\text { Attitude } \\
\text { Mean } \pm \text { SD }\end{array}$ & $p$ Value \\
\hline Sex & $\begin{array}{c}\text { Male } \\
\text { Female }\end{array}$ & $\begin{array}{l}3.15 \pm 0.46 \\
3.24 \pm 0.50\end{array}$ & 0.02 & $\begin{array}{l}3.24 \pm 0.53 \\
3.38 \pm 0.50\end{array}$ & 0.001 & $\begin{array}{l}3.19 \pm 0.48 \\
3.29 \pm 0.44\end{array}$ & 0.005 \\
\hline Degree & $\begin{array}{l}\text { Nursing } \\
\text { Physiotherapy } \\
\text { Chiropody }\end{array}$ & $\begin{array}{l}3.28 \pm 0.43 \\
3.07 \pm 0.51 \\
3.14 \pm 0.53\end{array}$ & $p<0.0001$ & $\begin{array}{l}3.41 \pm 0.45 \\
3.16 \pm 0.61 \\
3.29 \pm 0.35\end{array}$ & $p<0.0001$ & $\begin{array}{l}3.33 \pm 0.40 \\
3.10 \pm 0.51 \\
3.19 \pm 0.52\end{array}$ & $p<0.0001$ \\
\hline $\begin{array}{l}\text { Nursing } \\
\text { course year }\end{array}$ & $\begin{array}{l}\text { 1st } \\
\text { 2nd } \\
\text { 3rd } \\
4 \text { th }\end{array}$ & $\begin{array}{l}3.19 \pm 0.45 \\
3.29 \pm 0.42 \\
3.32 \pm 0.39 \\
3.42 \pm 0.42\end{array}$ & $p<0.0001$ & $\begin{array}{l}3.40 \pm 0.46 \\
3.43 \pm 0.43 \\
3.35 \pm 0.48 \\
3.50 \pm 0.41\end{array}$ & 0.125 & $\begin{array}{l}3.27 \pm 0.43 \\
3.34 \pm 0.38 \\
3.33 \pm 0.39 \\
3.45 \pm 0.39\end{array}$ & 0.005 \\
\hline $\begin{array}{l}\text { Physiotherapy } \\
\text { course year }\end{array}$ & $\begin{array}{l}\text { 1st } \\
\text { 2nd } \\
\text { 3rd } \\
4 \text { th }\end{array}$ & $\begin{array}{l}3.07 \pm 0.42 \\
3.02 \pm 0.48 \\
2.88 \pm 0.64 \\
3.26 \pm 0.45\end{array}$ & 0.015 & $\begin{array}{l}3.25 \pm 0.59 \\
3.10 \pm 0.60 \\
3.02 \pm 0.79 \\
3.23 \pm 0.48\end{array}$ & 0.356 & $\begin{array}{l}3.14 \pm 0.46 \\
3.05 \pm 0.49 \\
2.94 \pm 0.66 \\
3.25 \pm 0.43\end{array}$ & 0.070 \\
\hline $\begin{array}{l}\text { Chiropody } \\
\text { course year }\end{array}$ & $\begin{array}{l}\text { 1st } \\
\text { 2nd } \\
\text { 3rd } \\
4 \text { th }\end{array}$ & $\begin{array}{l}3.13 \pm 0.66 \\
3.13 \pm 0.48 \\
3.20 \pm 0.53 \\
3.12 \pm 0.50\end{array}$ & 0.947 & $\begin{array}{l}3.38 \pm 0.60 \\
3.25 \pm 0.55 \\
3.26 \pm 0.63 \\
3.29 \pm 0.50\end{array}$ & 0.767 & $\begin{array}{l}3.22 \pm 0.62 \\
3.17 \pm 0.48 \\
3.22 \pm 0.55 \\
3.18 \pm 0.45\end{array}$ & 0.964 \\
\hline
\end{tabular}

For 20 of the $24(83 \%)$ items in the questionnaire, over $70 \%$ of the students gave a positive response. For example, only $0.9 \%$ would not recommend the established vaccination schedule to their patients, and just $0.6 \%$ would not give their patients all the information on the effectiveness and possible adverse effects of a vaccine. However, $15.6 \%$ indicated that they did not consider it their ethical duty to be vaccinated against influenza, and $17.2 \%$ would not be vaccinated every year. Overall, the nursing students were those with the 
most positive beliefs and behaviour scores, followed by the physiotherapy and chiropody students (Table 4).

Table 4. ACVECS questionnaire: frequencies and percentages of students showing negative scores with respect to degree.

\begin{tabular}{|c|c|c|c|c|c|}
\hline \multirow[b]{2}{*}{ Questionnaire Items } & \multirow[b]{2}{*}{$\begin{array}{l}\text { Total } \\
n(\%)\end{array}$} & \multicolumn{4}{|c|}{ Degree } \\
\hline & & $\begin{array}{l}\text { Nursing } \\
n(\%)\end{array}$ & $\begin{array}{l}\text { Physiotherapy } \\
n(\%)\end{array}$ & $\begin{array}{c}\text { Chiropody } \\
n(\%)\end{array}$ & $p$ Value \\
\hline $\begin{array}{l}\text { 1.- I have doubts about the effectiveness of } \\
\text { vaccines }\end{array}$ & $104(11.1)$ & $78(12.5)$ & $12(10.5)$ & $14(9.5)$ & 0.341 \\
\hline $\begin{array}{l}\text { 2.- I would rather have influenza than be } \\
\text { vaccinated against it }\end{array}$ & $96(10.3)$ & $61(9.8)$ & $16(9.9)$ & 19 (12.8) & 0.736 \\
\hline $\begin{array}{l}\text { 3.- I am convinced that marketed vaccines are } \\
\text { safe }\end{array}$ & $73(7.8)$ & 44 (7.1) & 19 (11.7) & $10(6.8)$ & 0.067 \\
\hline $\begin{array}{l}\text { 4.- I am interested in learning more about } \\
\text { vaccination }\end{array}$ & 71 (7.6) & $31(5.0)$ & $17.3(12)$ & $12(8.1)$ & $p<0.001$ \\
\hline $\begin{array}{l}\text { 5.- I believe it important to check my vaccination } \\
\text { status before travelling to a tropical country such } \\
\text { as Mexico or Thailand }\end{array}$ & $7(0.7)$ & $4(0.6)$ & $1(0.6)$ & $2(1.4)$ & 0.210 \\
\hline $\begin{array}{l}\text { 6.- National and international vaccine campaigns } \\
\text { are cost-effective }\end{array}$ & $146(15.6)$ & $103(16.5)$ & 22 (13.6) & $21(11.2)$ & 0.296 \\
\hline $\begin{array}{l}\text { 7.- It is not worth being vaccinated against a } \\
\text { disease for which effective treatment exists }\end{array}$ & $56(6.0)$ & $33(5.3)$ & $16(9.9)$ & $7(4.7)$ & 0.006 \\
\hline $\begin{array}{l}\text { 8.- Vaccinating the adult population is not } \\
\text { important }\end{array}$ & $30(3.2)$ & $15(2.4)$ & $9(5.6)$ & $6(4.1)$ & 0.106 \\
\hline $\begin{array}{l}\text { 9.- Health science students are ethically obliged } \\
\text { to be vaccinated against influenza }\end{array}$ & $146(15.6)$ & 88 (14.1) & $34(21.0)$ & $24(16.2)$ & 0.147 \\
\hline $\begin{array}{l}\text { 10.- Being vaccinated myself has a positive } \\
\text { influence on the behaviour of my patients }\end{array}$ & $51(5.5)$ & $30(4.8)$ & $12(7.4)$ & $9(6.1)$ & $p<0.001$ \\
\hline $\begin{array}{l}\text { 11.- Students should be vaccinated to reduce the } \\
\text { transmission of infectious diseases in hospitals }\end{array}$ & $22(2.4)$ & $10(1.6)$ & $5(3.1)$ & $7(4.7)$ & 0.043 \\
\hline $\begin{array}{l}\text { 12.- I should review my vaccination status before } \\
\text { starting clinical training }\end{array}$ & $28(3.0)$ & $20(3.2)$ & $1(0.6)$ & $7(4.7)$ & 0.488 \\
\hline $\begin{array}{l}\text { 13.- I should be vaccinated against influenza } \\
\text { every year, even it means missing hours of } \\
\text { practical training }\end{array}$ & $159(17.0)$ & 81 (13.0) & $45(27.8)$ & $33(22.3)$ & $p<0.001$ \\
\hline $\begin{array}{l}\text { 14.- I would be vaccinated irrespective of what } \\
\text { my peers might do }\end{array}$ & $25(2.7)$ & $11(1.8)$ & $5(3.1)$ & $9(6.1)$ & 0.018 \\
\hline $\begin{array}{l}\text { 15.- If I am in good health there is no need to be } \\
\text { vaccinated }\end{array}$ & $55(5.9)$ & $33(5.3)$ & $12(7.4)$ & $10(6.8)$ & 0.002 \\
\hline $\begin{array}{l}\text { 16.- I would recommend my patients adhere to } \\
\text { the established vaccination calendar }\end{array}$ & $8(0.9)$ & $3(0.5)$ & $3(1.9)$ & $2(1.4)$ & $p<0.001$ \\
\hline $\begin{array}{l}\text { 17.- I would inform my patients of the } \\
\text { effectiveness, indications and side effects of each } \\
\text { vaccine }\end{array}$ & $6(0.6)$ & $1(0.2)$ & $4(2.5)$ & $1(0.7)$ & $p<0.001$ \\
\hline $\begin{array}{l}\text { 18.- I would travel to a tropical country only } \\
\text { after consulting Spain's International } \\
\text { Vaccination about the vaccines I require }\end{array}$ & $35(3.7)$ & $22(3.5)$ & $11(6.8)$ & $2(1.4)$ & 0.050 \\
\hline $\begin{array}{l}\text { 19.- I would be vaccinated against HIV when a } \\
\text { vaccine becomes available and when shown to } \\
\text { be acceptably safe and effective }\end{array}$ & $32(3.4)$ & $24(3.8)$ & $6(3.7)$ & $2(1.4)$ & 0.190 \\
\hline $\begin{array}{l}\text { 20.- If being vaccinated against influenza were } \\
\text { readily accessible to me I would be vaccinated } \\
\text { every year }\end{array}$ & $64(6.9)$ & $36(5.8)$ & $16(9.9)$ & $12(8.1)$ & 0.083 \\
\hline $\begin{array}{l}\text { 21.- I would be vaccinated against anything my } \\
\text { doctor recommends, even if I have to pay for it } \\
\text { 22.- When I begin work at a hospital I will make }\end{array}$ & $87(9.3)$ & $56(9.0)$ & $21(13.0)$ & $10(6.8)$ & 0.006 \\
\hline $\begin{array}{l}\text { sure I am vaccinated against everything } \\
\text { preventable }\end{array}$ & $10(1.1)$ & $3(0.5)$ & $3(1.9)$ & $4(2.7)$ & 0.029 \\
\hline $\begin{array}{l}\text { 23.- I would only be vaccinated in exceptional } \\
\text { circumstances (epidemics, health alerts etc. }\end{array}$ & $112(12.0)$ & 70 (11.2) & 25 (15.4) & 17 (11.5) & 0.333 \\
\hline $\begin{array}{l}\text { 24.- I will be vaccinated against influenza every } \\
\text { year I have clinical training }\end{array}$ & $122(13.1)$ & 67 (10.7) & $21(13.0)$ & $34(23.0)$ & $p<0.001$ \\
\hline
\end{tabular}


Multivariate analysis revealed female students, and to be following a nursing degree to be related to a more positive behaviour. Following a nursing degree and to be in more senior years of that degree was associated with a more positive score for beliefs. Female students, following a nursing degree, and being in the more senior years of that degree were also related to a better general attitude towards vaccination (Table 5).

Table 5. Multivariate analysis: relationships between beliefs, behaviour and general attitude and the variables sex, age degree being pursued and course year.

\begin{tabular}{|c|c|c|c|c|c|c|}
\hline Variables & $\begin{array}{c}\text { Beliefs } \\
\text { (B Coefficient) }\end{array}$ & $p$ Value & $\begin{array}{c}\text { Behaviour } \\
\text { (B Coefficient) }\end{array}$ & $p$ Value & $\begin{array}{c}\text { General } \\
\text { Attitude } \\
\text { (B Coefficient) }\end{array}$ & $p$ Value \\
\hline Sex & $\begin{array}{c}-0.07 \\
(-0.14,0.10)\end{array}$ & 0.088 & $\begin{array}{c}-0.11 \\
(-0.19,-0.03)\end{array}$ & 0.008 & $\begin{array}{c}-0.08 \\
(-0.16,-0.01)\end{array}$ & 0.026 \\
\hline Age & $\begin{array}{c}-0.003 \\
(-0.009,0.002)\end{array}$ & 0.267 & $\begin{array}{c}-0.002 \\
(-0.006,0.006)\end{array}$ & 0.984 & $\begin{array}{c}-0.002 \\
(-0.008,0.004)\end{array}$ & 0.469 \\
\hline Degree & $\begin{array}{c}-0.10 \\
(-0.14,-0.05)\end{array}$ & $p<0.001$ & $\begin{array}{c}-0.09 \\
(-0.13,-0.04)\end{array}$ & $p<0.001$ & $\begin{array}{c}-0.09 \\
(-0.13,-0.05)\end{array}$ & $p<0.001$ \\
\hline Course year & $\begin{array}{c}0.06 \\
(0.03,0.09)\end{array}$ & $p<0.001$ & $\begin{array}{c}0.002 \\
(-0.030,0.033)\end{array}$ & 0.916 & $\begin{array}{c}0.04 \\
(0.01-0.07)\end{array}$ & 0.009 \\
\hline
\end{tabular}

In this case, 250 students (26.8\%) declared having been vaccinated against influenza in the campaign preceding the study; no significant difference was seen between male (25.2\%) and female $(27.2 \%)$ students. In total, $32.5 \%$ of the nursing students were vaccinated, $26.8 \%$ of the chiropody students, and $17.9 \%$ of the physiotherapy students $(p<0.001)$. In terms of course year, second year students were those who most often sought vaccination $(40.3 \%)$, followed by third year $(31.5 \%)$, fourth year $(21.3 \%)$, and first year $(13.0 \%)$ students $(p<0.001)$. Those students who scored positive behaviour scores sought vaccination more often than those with poorer scores $(28.7 \%$ vs. $13.4 \% ; p<0.001)$.

The likelihood of seeking vaccination increased with age (OR 1.04 [1.01-1.07]). The nursing students were more likely to have sought vaccination to influenza than either the physiotherapy (OR 2.61 [1.30-5.21]) or chiropody (OR 4.53 [2.57-7.96] students. Those students with positive belief scores were also more likely to have sought vaccination than those with poorer scores (OR 2.06 [1.18-3.60]); the same was seen for those students with positive behaviour scores (OR 3.74 [2.17-6.44]) (Table 6).

Table 6. Bivariate and multivariate analyses: effect of sex, age, degree being pursued, course year, beliefs and behaviour on seeking vaccination to influenza.

\begin{tabular}{|c|c|c|c|c|c|c|}
\hline Variables & $\beta$ & OR (95CI\%) & $p$ Value & $\beta$ & OR $(95 \mathrm{CI} \%)$ & $p$ Value \\
\hline \multicolumn{7}{|l|}{ Sex } \\
\hline females vs. males & 0.10 & $\begin{array}{c}1.11 \\
(0.77-1.60)\end{array}$ & 0.567 & & & \\
\hline Age & 0.03 & $\begin{array}{c}1.03 \\
(1.00-1.05)\end{array}$ & 0.029 & 0.04 & $\begin{array}{c}1.04 \\
(1.01-1.07)\end{array}$ & 0.006 \\
\hline \multicolumn{7}{|l|}{ Degree } \\
\hline nursing vs. physiotherapy & 0.79 & $\begin{array}{c}2.21 \\
(1.43-3.42)\end{array}$ & $p<0.001$ & 0.96 & $\begin{array}{c}2.61 \\
(1.30-5.21)\end{array}$ & 0.007 \\
\hline nursing vs. chiropody & 1.25 & $\begin{array}{c}3.48 \\
(2.07-5.86)\end{array}$ & $p<0.001$ & 1.51 & $\begin{array}{c}4.53 \\
(2.57-7.96)\end{array}$ & $p<0.001$ \\
\hline \multicolumn{7}{|l|}{ Course year } \\
\hline 2nd vs. 1st & 1.51 & $\begin{array}{c}4.52 \\
(2.99-6.82)\end{array}$ & $p<0.001$ & 1.82 & $\begin{array}{c}6.20 \\
(3.96-9.71)\end{array}$ & $p<0.001$ \\
\hline 3rd vs. 1st & 1.12 & $\begin{array}{c}3.07 \\
(1.93-4.89)\end{array}$ & $p<0.001$ & 1.30 & $\begin{array}{c}3.65 \\
(2.21-6.03)\end{array}$ & $p<0.001$ \\
\hline
\end{tabular}


Table 6. Cont.

\begin{tabular}{ccccccc}
\hline Variables & $\beta$ & OR (95CI\%) & $p$ Value & $\beta$ & OR (95CI\%) & $p$ Value \\
\hline 4th vs. 1st & 0.60 & $\begin{array}{c}1.81 \\
(1.09-3.01)\end{array}$ & $p=0.021$ & 0.52 & $\begin{array}{c}1.68 \\
(0.96-2.91)\end{array}$ & $\begin{array}{c}p=0.067 \\
(1.06-3.60)\end{array}$ \\
\hline Beliefs & 1.55 & $\begin{array}{c}4.73 \\
(3.2-6.99)\end{array}$ & $p<0.001$ & 0.72 & $\begin{array}{c}3.011 \\
(2.17-6.44)\end{array}$ \\
\hline Behaviour & 1.59 & $\begin{array}{c}4.89 \\
(3.32-7.22)\end{array}$ & $p<0.001$ & 1.32 & & \\
\hline General attitude & 1.85 & $\begin{array}{c}6.33 \\
(4.11-9.76)\end{array}$ & $p<0.001$ & & \\
\hline
\end{tabular}

\section{Discussion}

A total of 934 students completed the questionnaire- $59.1 \%$ of the faculty's entire student body. This is a high percentage compared to similar studies [22-25].

In general, the present students had a good general attitude towards vaccination. Good scores were returned for all the dimensions examined; indeed, they were higher than those obtained in the study in which the questionnaire was originally validated [22]. Studies similar to the present performed in Serbia [23], Florida (USA) [26] and Australia [27] obtained even more positive results, while others performed in the USA [25], Germany [28], Canada [24] and Italy [29] recorded fewer positive results.

In the present work, female students were associated with a more positive general attitude towards vaccination; this has also been reported from the USA [30], Poland [31] and a previous study undertaken in Spain [32]. In contrast, an Italian study found male nursing students to have a better attitude [33]. It is not clear why women have a more positive attitude towards vaccination than men.

The nursing students returned better beliefs, behaviour and general attitude scores than did the students pursuing the other degrees. This might be explained in that nurses have far more responsibility than physiotherapists or chiropodists viz a viz vaccine administration [34], and that their studies include much more extensive content on immunisation. Even though the present study did not examine student knowledge of vaccination, the literature contains ample evidence of a correlation between level of knowledge and attitude towards vaccination $[23,24,26]$. The better general attitude shown by the students of nursing compared to those of other professions could not, however, be confirmed by the literature; no studies were found assessing the attitudes of physiotherapy and chiropody students. Some studies show students of nursing to be less knowledgeable and to have a poorer attitude towards vaccination than students of medicine and pharmacy [24,25].

The general attitude of the nursing students became better year-on year as their course progressed. This has been reported previously in two Spanish studies [22,32] and other international studies [23,35]. This is probably due to their increased knowledge in their more senior years, reflecting the above-mentioned relationship between knowledge and attitude.

Even though both Spanish [36] and international bodies [37] recommend vaccination against influenza for students of health sciences, only $26.7 \%$ of the 934 respondents had been so vaccinated. This is lower than that reported from the USA [38] and Australia [39], similar to that recorded in Israel [40] and Ireland [41], and very much higher than that reported in studies from China [42], Italy [43], Poland [44] and indeed from another study performed in Spain [45].

In agreement with other studies, multivariate analysis showed that female students [45], increasing age [42,45], studying nursing rather than physiotherapy $[39,44]$, and having more positive beliefs and behaviour $[38,41,42]$ to be associated with seeking vaccination against influenza.

The low coverage of influenza vaccination among the chiropody students $(12.2 \%$ compared to $32.5 \%$ among nursing students and $17.9 \%$ among physiotherapy students) 
might be influenced by the lesser content on vaccination in their course. In addition, the chiropody students do not undertake their practical training at external health centres but at the University's chiropody clinic. Similarly, the finding that the second-year nursing students had the highest coverage might be explained by their training at external health centres at the time of the yearly influenza vaccination campaign.

It is important to note that the present results were not influenced by the COVID-19 pandemic since they were collected before it began in Spain. Even though news items on the disease were published at that time, the present high level of media attention had by no means been reached. It would be of interest to repeat this study when the pandemic is over.

The present work suffers the limitation that the study subjects were only those willing to take part who were present on the days when the project was explained. Even though those who responded represented $59.1 \%$ of the entire student body, no information was collected on those who did not participate. It remains possible that their characteristics were different to those who took part.

Finally, the present results were all obtained from health science students at one university; they may not be extrapolatable to those attending other universities.

\section{Conclusions}

The beliefs, behaviour and general attitude towards vaccination among the study subjects can be said to be positive, especially among the students of nursing, and they improve year on year as these students progress through their course. These findings are encouraging since it is largely nurses who are responsible for administering vaccines; they also reflect a positive influence of the instruction they receive. Even though physiotherapists and chiropodists are not at the forefront of vaccination campaigns, they are health professionals, and it might be useful to increase the content on vaccination in their courses.

The coverage of vaccination against influenza, while better than that seen in some studies and similar to that recorded in others, was still quite low. Debate is required on how to increase this coverage among health science students, especially among those undertaking training periods at health centres. The finding that those students with better beliefs and behaviour scores were those among whom this coverage was greatest reflects a need to design strategies that improve the general attitude towards vaccination of those students with lower scores.

Author Contributions: Conceptualisation and methodology: P.-R.F.J., E.-G.A. and D.G.-L.R.; validation, formal analysis and writing-original draft preparation: P.-R.F.J. and A.B.M.J.; research, writing-review, editing and visualisation: P.-R.F.J., E.-G.A., D.G.-L.R., M.-V.C.M.A. and A.B.M.J. All authors have read and agreed to the submitted version of the manuscript.

Funding: This work was funded by the Universidad Complutense de Madrid via the 'Ayudas Económicas a los Grupos de Investigación UCM (Aplicación presupuestaria G/6400100/3000)’

Institutional Review Board Statement: The study was conducted according to the guidelines of the Declaration of Helsinki, and approved by the Ethics in Research Committee of the Hospital Universitario Clínico San Carlos (protocol code 20/376-E and date of approval: 7 May 2021).

Informed Consent Statement: Informed consent was obtained from all subjects involved in the study. Written informed consent has been obtained from the patient(s) to publish this paper.

Acknowledgments: Thanks are extended to the coordinators and teachers of the Nursing, Physiotherapy and Chiropody degree courses for their assistance in this project, and to the anonymous students who completed the questionnaire.

Conflicts of Interest: The authors declare no conflict of interest. 


\section{References}

1. Andre, E.; Booy, R.; Bock, H.L.; Clemens, J.; Datta, S.K.; John, T.J.; Lee, B.W.; Lolekha, S.; Peltola, H.; A Ruff, T.; et al. Vaccination greatly reduces disease, disability, death and inequity worldwide. Bull. World Health Organ. 2008, 86, 140-146. [CrossRef]

2. Remy, V.; Zöllner, Y.; Heckmann, U. Vaccination: The cornerstone of an efficient healthcare system. J. Mark. Access Health Policy 2015, 3, 27041. [CrossRef]

3. Ethgen, O.; Rémy, V.; Wargo, K. Vaccination budget in Europe: An update. Hum. Vaccin Immunother. 2018, 14, 2911-2915. [CrossRef] [PubMed]

4. Ministerio de Sanidad Consumo y Bienestar Social. Los sistemas sanitarios en los países de la Unión Europea (UE). In Características e Indicadores de Salud 2019; Secretaría General de Sanidad y Consumo: Madrid, Germany, 2019. Available online: http:/ / sanidadue.es/es/portada (accessed on 9 October 2020).

5. Dubé, E.; Vivion, M.; MacDonald, E.N. Vaccine hesitancy, vaccine refusal and the anti-vaccine movement: Influence, impact and implications. Expert Rev. Vaccines 2015, 14, 99-117. [CrossRef] [PubMed]

6. Larson, S.; de Figueiredo, A.; Karafillakis, E.; Rawal, M. The State of Vaccine Confidence in the EU 2018; London School of Hygiene and Tropical Medicine: Luxemburg, 2018. Available online: https://ec.europa.eu/health/sites/health/files/vaccination/docs/ 2018_vaccine_confidence_en.pdf (accessed on 9 October 2020).

7. World Health Organization. Report of the Strategic Advisory Group of Experts Working Group on Vaccine Hesitancy; WHO: Ginebra, Swizterland, 2014. Available online: https://www.who.int/immunization/sage/meetings/2014/october/1_Report_WORKING_ GROUP_vaccine_hesitancy_final.pdf (accessed on 25 November 2020).

8. European Centre for Disease Prevention and Control. Let's Talk about Hesitancy: Enhancing Confidence in Vaccination and Uptake. Practical Guide for Public Health Programme Managers and Communicators; ECDC: Stockholm, Sweden, 2016. Available online: https://www.ecdc.europa.eu/sites/portal/files/media/en/publications/Publications/lets-talk-about-hesitancyvaccination-guide.pdf (accessed on 25 November 2020).

9. European Centre for Disease Prevention and Control. Vaccine Hesitancy among Healthcare Workers and Their Patients in Europe-A Qualitative Study; ECDC: Stockholm, Sweden, 2015. Available online: https:/ / www.ecdc.europa.eu/sites/default/files / media/ en/publications/Publications/vaccine-hesitancy-among-healthcare-workers.pdf (accessed on 26 November 2020).

10. World Health Organization. Diez Cuestiones de Salud Que la OMS Abordará Este Año; WHO: Geneva, Swizterland, 2019. Available online: https:/ / www.who.int/es/news-room/feature-stories/ten-threats-to-global-health-in-2019 (accessed on 26 November 2020).

11. Dubé, E. Addressing vaccine hesitancy: The crucial role of healthcare providers. Clin. Microbiol. Infect. 2017, 23, 279-280. [CrossRef]

12. Herzog, R.; Álvarez-Pasquin, M.J.; Díaz, C.; Del Barrio, J.L.; Estrada, J.M.; Gil, Á. Are healthcare workers' intentions to vaccinate related to their knowledge, beliefs and attitudes? A systematic review. BMC Public Health 2013, 13, 154. [CrossRef] [PubMed]

13. Yaqub, O.; Castle-Clarke, S.; Sevdalis, N.; Chataway, J. Attitudes to vaccination: A critical review. Soc. Sci. Med. 2014, 112, 1-11. [CrossRef]

14. Paterson, P.; Meurice, F.; Stanberry, L.R.; Glismann, S.; Rosenthal, S.L.; Larson, H.J. Vaccine hesitancy and healthcare providers. Vaccine 2016, 34, 6700-6706. [CrossRef] [PubMed]

15. Schmitt, H.-J.; Booy, R.; Aston, R.; Van Damme, P.; Schumacher, R.F.; Campins, M.; Rodrigo, C.; Heikkinen, T.; Weil-Olivier, C.; Finn, A.; et al. How to optimise the coverage rate of infant and adult immunisations in Europe. BMC Med. 2007, 5, 11. [CrossRef]

16. O'Leary, S.T.; Allison, M.A.; Stokley, S.; Crane, L.A.; Hurley, L.P.; Beaty, B.; Kempe, A. Physicians' confidence in vaccine safety studies. Prev. Med. 2013, 56, 231-233. [CrossRef]

17. Picchio, C.A.; Carrasco, M.G.; Sagué-Vilavella, M.; Rius, C. Knowledge, attitudes and beliefs about vaccination in primary healthcare workers involved in the administration of systematic childhood vaccines, Barcelona, 2016/17. Euro. Surveill. 2019, 24, 1800117. [CrossRef] [PubMed]

18. Hough-Telford, C.; Kimberlin, D.W.; Aban, I.; Hitchcock, W.P.; Almquist, J.; Kratz, R.; O'Connor, K.G. Vaccine Delays, Refusals, and Patient Dismissals: A Survey of Pediatricians. Pediatrics 2016, 138, e20162127. [CrossRef] [PubMed]

19. Berry, N.J.; Henry, A.; Danchin, M.; Trevena, L.J.; Willaby, H.W.; Leask, J. When parents won't vaccinate their children: A qualitative investigation of australian primary care providers' experiences. BMC Pediatr. 2017, 17, 19. [CrossRef] [PubMed]

20. Suryadevara, M.; Handel, A.; Bonville, C.A.; Cibula, D.A.; Domachowske, J. Pediatric provider vaccine hesitancy: An underrecognized obstacle to immunizing children. Vaccine 2015, 33, 6629-6634. [CrossRef] [PubMed]

21. Mergler, M.J.; Omer, S.B.; Pan, W.K.; Navar-Boggan, A.M.; Orenstein, W.; Marcuse, E.K.; Taylor, J.; Dehart, M.P.; Carter, T.C.; Damico, A.; et al. Association of vaccine-related attitudes and beliefs between parents and health care providers. Vaccine 2013, 31, 4591-4595. [CrossRef]

22. Fernández-Prada, M.; Ramos-Martín, P.; Madroñal-Menéndez, J.; Martínez-Ortega, C.; González-Cabrera, J. Diseño y validación de un cuestionario sobre vacunación en estudiantes de ciencias de la salud. Rev. Esp. Salud Publica 2016, 7, e1-e10.

23. Cvjetkovic, S.J.; Jeremic, V.L.; Tiosavljevic, D.V. Knowledge and attitudes toward vaccination: A survey of Serbian students. J. Infect. Public Health 2017, 10, 649-656. [CrossRef]

24. Pelly, L.P.; MacDougall, D.M.P.; A Halperin, B.; Strang, A.R.; Bowles, S.K.; Baxendale, D.M.; McNeil, A.S. The Vaxed Project: An Assessment of Immunization Education in Canadian Health Professional Programs. BMC Med. Educ. 2010, 10, 86. [CrossRef] 
25. Dybsand, L.L.; Hall, K.J.; Carson, P.J. Immunization attitudes, opinions, and knowledge of healthcare professional students at two Midwestern universities in the United States. BMC Med. Educ. 2019, 19, 242. [CrossRef]

26. Berera, D.; Thompson, K.M. Medical Student Knowledge, Attitudes, and Practices Regarding Immunization. J. Vaccines Vaccin. 2012, 6, 2. [CrossRef]

27. Walker, L.; Newall, A.; Heywood, A.E. Knowledge, attitudes and practices of Australian medical students towards influenza vaccination. Vaccine 2016, 34, 6193-6199. [CrossRef]

28. Lehmann, A.B.; Ruiter, R.A.C.; Wicker, S.; Chapman, G.; Kok, G. Medical students' attitude towards influenza vaccination. BMC Infect. Dis. 2015, 15, 185. [CrossRef]

29. La Fauci, V.; Squeri, R.; Genovese, C.; Anzalone, C.; Fedele, F.; Squeri, A.; Alessi, V. An observational study of university students of healthcare area: Knowledge, attitudes and behaviour towards vaccinations. Clin Ter. 2019, 170, e448-e453. [CrossRef]

30. Afonso, N.M.; Kavanagh, M.J.; Swanberg, S.M.; Schulte, J.M.; Wunderlich, T.; Lucia, V.C. Will they lead by example? Assessment of vaccination rates and attitudes to human papilloma virus in millennial medical students. BMC Public Health 2017, 17, 35. [CrossRef] [PubMed]

31. Zarobkiewicz, M.K.; Zimecka, A.; Zuzak, T.; Cieślak, D.; Roliński, J.; Grywalska, E. Vaccination among Polish university students. Knowledge, beliefs and anti-vaccination attitudes. Hum. Vaccines Immunother. 2017, 13, 2654-2658. [CrossRef] [PubMed]

32. Villanueva, S.; Mosteiro-Miguéns, D.G.; Domínguez-Martís, E.M.; López-Ares, D.; Novío, S. Knowledge, Attitudes, and Intentions towards Human Papillomavirus Vaccination among Nursing Students in Spain. Int. J. Environ. Res. Public Health 2019, $16,4507$. [CrossRef] [PubMed]

33. Pelullo, C.P.; Esposito, M.R.; Di Giuseppe, G. Human Papillomavirus Infection and Vaccination: Knowledge and Attitudes among Nursing Students in Italy. Int. J. Environ. Res. Public Health 2019, 16, 1770. [CrossRef]

34. Forcada, J.A. El acto vacunal. Vacunas 2014, 15, 272-283. [CrossRef]

35. Shrestha, R.; Chhettri, P.; Bhusal, C.; Ruchal, R.; Shrestha, S.; Shrestha, B. Knowledge, Attitude and Practice Regarding Immunization among Medical Students. JUCMS 2019, 7, 46-50.

36. de Ministerio, S. Vacunación Frente a la Gripe. Ministerio de Sanidad, Consumo y Bienestar Social: Madrid, Germany. Available online: https://www.mscbs.gob.es/profesionales/saludPublica/prevPromocion/vacunaciones/Vacunacion_Gripe.htm (accessed on 27 November 2020).

37. Grohskopf, L.A.; Alyanak, E.; Broder, K.R.; Walter, E.B.; Fry, A.M.; Jernigan, D.B. Prevention and Control of Seasonal Influenza with Vaccines: Recommendations of the Advisory Committee on Immunization Practices—United States, 2019-2020 Influenza Season. MMWR. Recomm. Rep. 2019, 68, 1-21. [CrossRef]

38. Rogers, C.J.; Bahr, K.O.; Benjamin, S.M. Attitudes and barriers associated with seasonal influenza vaccination uptake among public health students; a cross-sectional study. BMC Public Health 2018, 18, 1131. [CrossRef] [PubMed]

39. Kelly, D.A.; Macey, D.J.; Mak, D.B. Annual influenza vaccination. Hum. Vaccines Immunother. 2014, 10, 1930-1934. [CrossRef]

40. Shahrabani, S.; Benzion, U.; Din, G.Y. Factors affecting nurses' decision to get the flu vaccine. Eur. J. Health Econ. 2008, 10, 227-231. [CrossRef] [PubMed]

41. Cornally, N.; Deasy, E.A.; McCarthey, G.; McAuley, C.; Moran, J.; Weathers, E. Student nurses' intention to get the influenza vaccine. Br. J. Nurs. 2013, 22, 1207-1211. [CrossRef] [PubMed]

42. Cheung, K.; Ho, S.M.S.; Lam, W. Factors affecting the willingness of nursing students to receive annual seasonal influenza vaccination: A large-scale cross-sectional study. Vaccine 2017, 35, 1482-1487. [CrossRef]

43. Bonaccorsi, G.; Lorini, C.; Porchia, B.R.; Niccolai, G.; Martino, G.; Giannarelli, L.; Santomauro, F. Influenza vaccination: Coverage and risk perception among students of the health professions at Florence University, Italy. Ann. Ig. 2013, 25, 181-189. [PubMed]

44. Woźniak-Kosek, A.; Kempińska-Mirosławska, B.; Mendrycka, M.; Saracen, A.; Hoser, G. Factors affecting decision concerning influenza vaccination among students of medical faculties. Acta Biochim. Pol. 2014, 61, 829-832. [CrossRef]

45. Hernández-García, I.; Cardoso-Muñoz, A.M.; Valero-Juan, L.F.; Giménez-Júlvez, M.T. Vacunación antigripal en estudiantes de enfermería durante la temporada 2014-2015. Rev. Esp. Salud Publica 2015, 89, 615-625. [CrossRef] 